\title{
NEREIDIDAE (POLYCHAETA) DA COSTA NORDESTE DO BRASIL. III. GÊNEROS CERATONEREIS E NEREIS
}

Cinthya S. G. Santos ${ }^{1}$ Paulo da Cunha Lana ${ }^{1}$

\begin{abstract}
NEREIDIDAE (POLYCHAETA) FROM NORTHEASTERN COAST OF THE BRAZIL. III. CERATONEREIS AND NEREIS. Ten species belonging to the genera Ceratonereis Kinberg, 1866 and Nereis Linnaeus, 1758 were recorded in estuaries, exposed sandy beaches, shelly soft bottoms, atolls and coral reefs of the Brazilian northeastern coast. Two new species, Nereis serrata, from Ceará coast, and Nereis pseudomoniliformis, from Sergipe coast, are described.
\end{abstract}

KEYWORDS. Annelida, Nereididae, taxonomy, northeastern Brazil.

\section{INTRODUÇÃO}

A família Nereididae é uma das mais conhecidas e com maior número de espécies descritas entre os poliquetas, com cerca de 45 espécies registradas ao longo da costa brasileira. Das 65 espécies do gênero Ceratonereis Kinberg, apenas cinco foram previamente registradas na costa brasileira (GRÜBE, 1874; HARTMAN, 1948; RULLIER \& Amoureux, 1979; Perkins, 1980; Morgado et al., 1994; SANTA-Isabel et al., 2000), e duas destas no nordeste. Das 85 espécies do gênero Nereis Linnaeus, seis foram identificadas no Brasil (Hansen, 1882; Treadwell, 1932; Nonato \& Luna, 1970; Rullier \& AmoureuX, 1979; Lana \& Sovierzoski, 1987; Paiva, 1993; Pagliosa \& Lana, 2000), quatro delas ocorrentes na costa nordeste.

O objetivo é realizar o levantamento das espécies de Ceratonereis e Nereis na costa nordeste do Brasil.

1. Centro de Estudos do Mar, Universidade Federal do Paraná, Caixa Postal 50002, 83 255-000, Pontal do Paraná, PR, Brasil. 


\section{MATERIAL E MÉTODOS}

Os espécimes provenientes da plataforma continental foram obtidos a partir dos Projetos Geocosta/1994, Ceará, Projeto Algas/1980, Paraíba e Projeto REVIZEE. Amostras provenientes da região sublitoral estuarina foram obtidas utilizando-se um pegador de fundo do tipo "Van Veen" (HoLmE \& MCINTYRE, 1971). As coleções provenientes da região entre marés, que inclui praias arenosas, arenolodosas, rochosas, manguezais, costões e recifes, foram obtidas através de testemunhadores e por raspagem do material incrustado. $\mathrm{O}$ material foi triado por meio de peneiras com malhas de $1,0 \mathrm{~mm}$ e $0,5 \mathrm{~mm}$, fixado em solução de formalina $4 \%$ e conservado em álcool $70 \%$. Alguns espécimes foram anestesiados com solução de cloreto de magnésio a $8 \%$. Os procedimentos taxonômicos são os adotados por DAY (1967) e a nomenclatura utilizada para caracterização morfológica, conforme DAY (1967) e Hutchings \& TuRvey (1982). A largura do corpo, com e sem parapódios, foi tomada no setígero 5. O material examinado encontra-se depositado na coleção de poliquetas do Centro de Estudos do Mar, Universidade Federal do Paraná (MCEM).

\section{Ceratonereis Kinberg, 1866}

Chave para as espécies de Ceratonereis da costa nordeste do Brasil.

1. Prostômio inteiro na região anterior ...... 2

Prostômio fendido na região anterior

2. Notocerdas espinígeras homogonfas ao longo do corpo ... C. hircinicola (Eisig, 1870)

Notocerdas sesquigonfas falcígeras nos parapódios medianos e posteriores

C. vittata Langerhans, 1884

3. Cerdas neuropodiais falcígeras heterogonfas bidentadas presentes em parapódios posteriores C. mirabilis Kinberg, 1866

Cerdas neuropodiais falcígeras heterogonfas bidentadas ausentes

4. Antenas foliáceas; lígulas notopodiais nos parapódios posteriores, podem ser vestigiais; tufos de pêlos das cerdas falcígeras heterogonfas ultrapassando distintamente o ápice do artículo

C. singularis Treadwell, 1929

Antenas cirriformes; lígulas notopodiais ausentes em parapódios posteriores; estruturas glandulares subdermais no prostômio; pêlos das falcígeras ultrapassam ligeiramente o ápice do artículo C. excisa (Grübe, 1874)

\section{Ceratonereis mirabilis Kinberg, 1866}

(Figs. 1-6)

Ceratonereis mirabilis Kinberg, 1866:170; Perkins, 1980:4, figs.1-4; Rullier \& Amoureux, 1979:108; TAYLOR, 1984:30, figs. 27a, 28 a-e.

Comentários. A espécie foi redescrita por Perkins (1980), que revisou todas as espécies semelhantes a C. mirabilis. Diferencia-se das quatro espécies co-genéricas registradas na costa brasileira pelo prostômio fortemente fendido, pelas notocerdas falcígeras bidentadas (figs. 4-6) e pelas lígulas notopodiais presentes em todos os parapódios (figs. 1-3).

Habitat. Em poças de maré com substrato calcário.

Distribuição. Oceano Índico: Ilhas Andaman, Madagáscar e África do Sul; Oceano Pacífico: Ilhas Galápagos e Japão; Atlântico Ocidental: Flórida, Golfo do México, Bermudas, Bahamas, Porto Rico, Barbados, Cuba e Brasil; Atlântico Oriental, Mar Vermelho.

Material examinado. BRASIL, Rio Grande do Norte: Atol das Rocas (MCEM 1125, 1126, 2 exs.). 


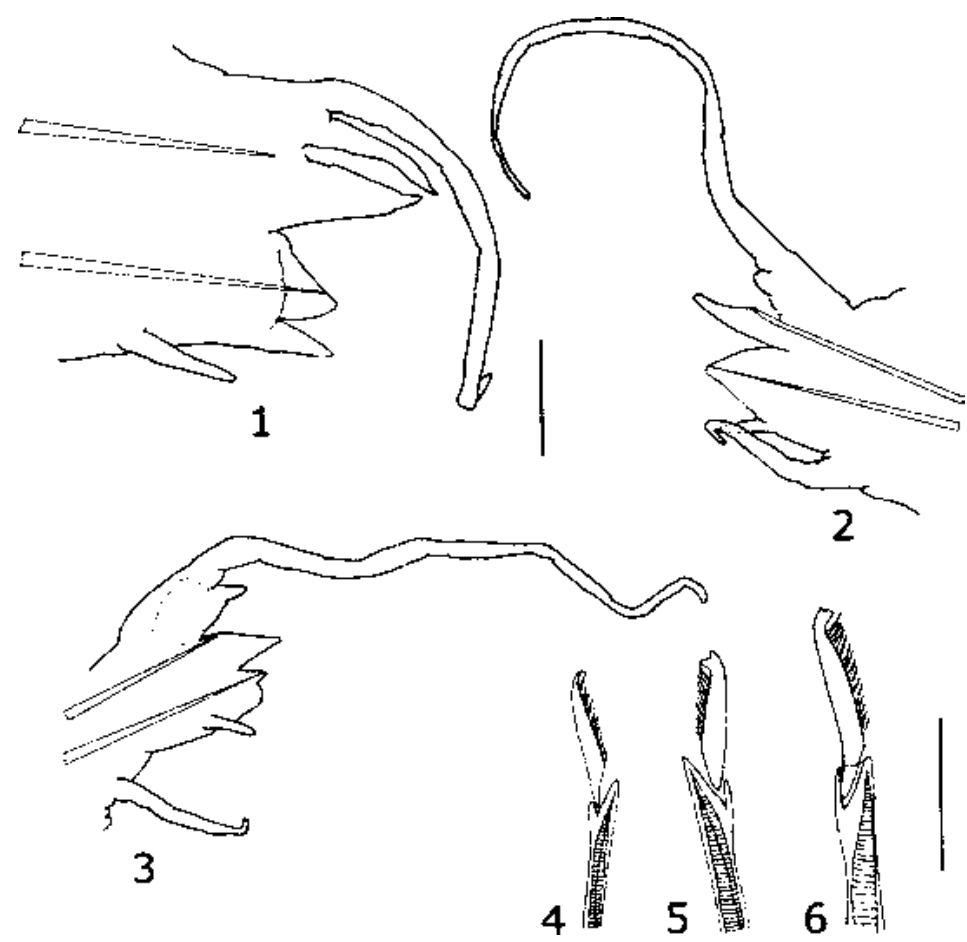

Figs. 1-6. Ceratonereis mirabilis Kinberg, 1866, Atol das Rocas, Rio Grande do Norte: 1, parapódio anterior, vista anterior; 2, parapódio mediano, vista anterior; 3, parapódio posterior, vista posterior; 4, cerda falcígera heterogonfa; 5 , cerda falcígera heterogonfa bidentada; 6 , cerda falcígera sesquigonfa bidentada. Escala: $0,5 \mathrm{~mm}$.

\section{Ceratonereis excisa (Grübe, 1874)}

(Figs. 7-12)

Nereis excisa GRÜBE, 1874:72.

Nereis (Ceratonereis) tentaculata; Augener, 1913:168.

Ceratonereis excisa; Perkins, 1980:15, fig. 6.

Comentários. Este registro amplia a distribuição da espécie cerca de $10^{\circ}$ para o norte. Ceratonereis excisa foi redescrita por PERKINS (1980) e caracteriza-se pelas estruturas prostomiais subdermais (fig. 7), ausência de lígulas notopodiais em parapódios posteriores (figs. 8-10) e cerdas falcígeras unidentadas (figs. 11,12). Os espécimes coletados no nordeste não apresentam variações significativas entre si ou em relação ao material revisado por PERKINS (1980). A presença de glândulas subdermais no prostômio, considerada diagnóstica de C. excisa, é igualmente atribuída a outras espécies (HARTMANN-SCHRÖDER, 1985).

Habitat. Região entre marés de estuários e praias.

Distribuição. Atlântico Ocidental: Brasil (Paraíba, Sergipe, Bahia e Santa Catarina).

Material examinado. BRASIL, Paraíba: João Pessoa,Tambaú (MCEM 1133-1135, 4 exs.); Estuário do rio Paraíba (MCEM 1136, 1 ex.); Sergipe: Estuário do rio Piauí (MCEM 1132, 1 ex.; MCEM 1137, 2 exs.); Bahia: Litoral Norte (MCEM 1138, 4 exs.). 


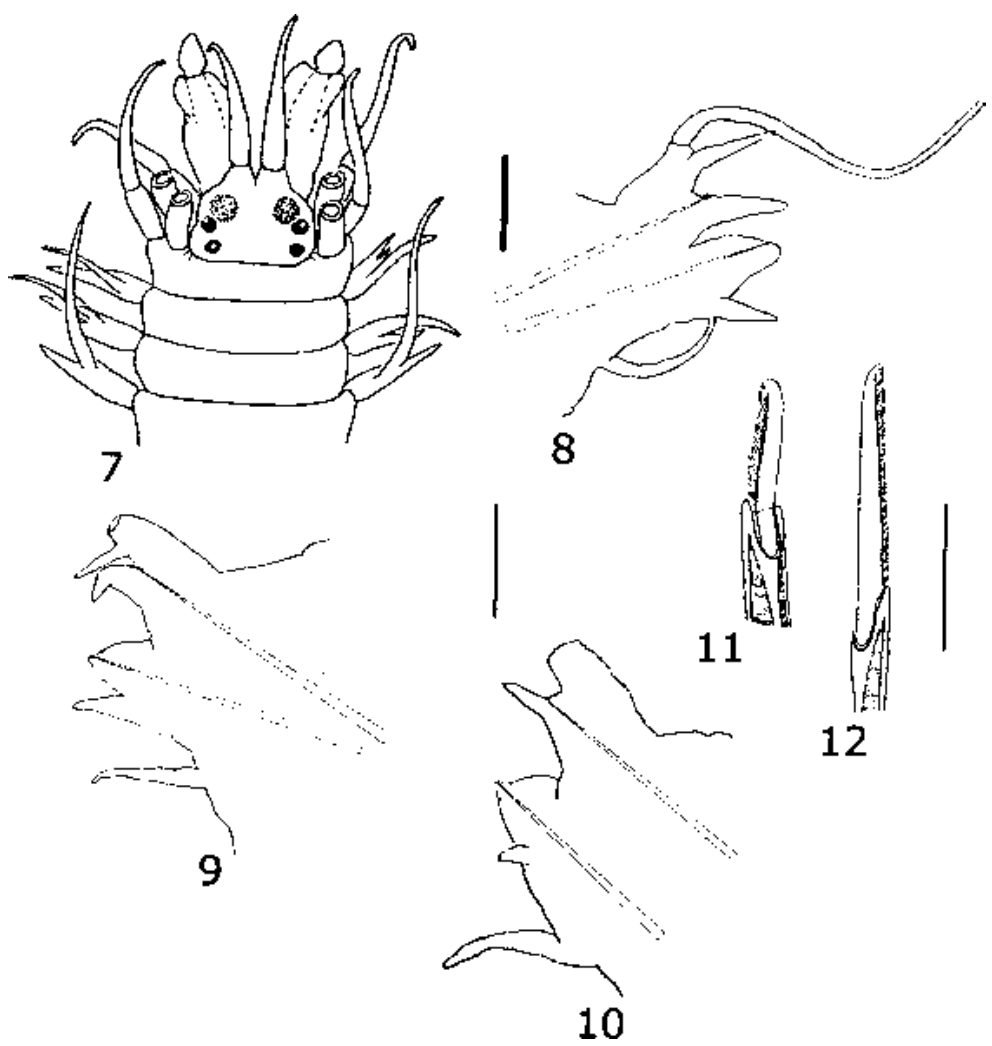

Figs. 7-12. Ceratonereis excisa (Grübe, 1874), Rio Piauí, Sergipe: 7, região anterior, vista dorsal; 8 , parapódio 5 , vista posterior; 9 , parapódio 30 , vista anterior; 10 , parapódio 68 , vista anterior; 11 , cerda falcígera sesquigonfa; 12 , cerda falcígera heterogonfa. Escalas: $0,5 \mathrm{~mm}$, fig. 7;0,1 mm, figs. 8-10;0,05 mm, figs. 11,12 .

\section{Ceratonereis hircinicola (Eisig, 1870)}

(Figs. 13-18)

Nereis hircinicola EISIG, 1870:103.

Ceratonereis hircinicola; DAy, 1967:327 [em parte]; IMAJIMA, 1972:68, fig. 14 m; Rullier \& Amoureux, 1979:168.

Comentários. A espécie foi descrita originalmente para o Mediterrâneo e tem ampla distribuição, tendo sido previamente identificada na região nordeste por RULLIER \& Amourex (1979). Ceratonereis hircinicola (fig. 13) difere de C. costae (Grübe, 1840), espécie similar, já referida para a costa brasileira, e de C. keiskama Day, 1953 pelo lóbulo notopodial bilobado. Espécimes identificados por DAY (1967) como C. hircinicola apresentam número de paragnatas muito maior do que o registrado por FAUVEL (1923) e IMAJIMA (1972), este último inclusive questiona aquela identificação. Os exemplares provenientes da costa nordeste assemelham-se àqueles descritos por esses dois autores, tanto no número de paragnatas como na morfologia parapodial. 

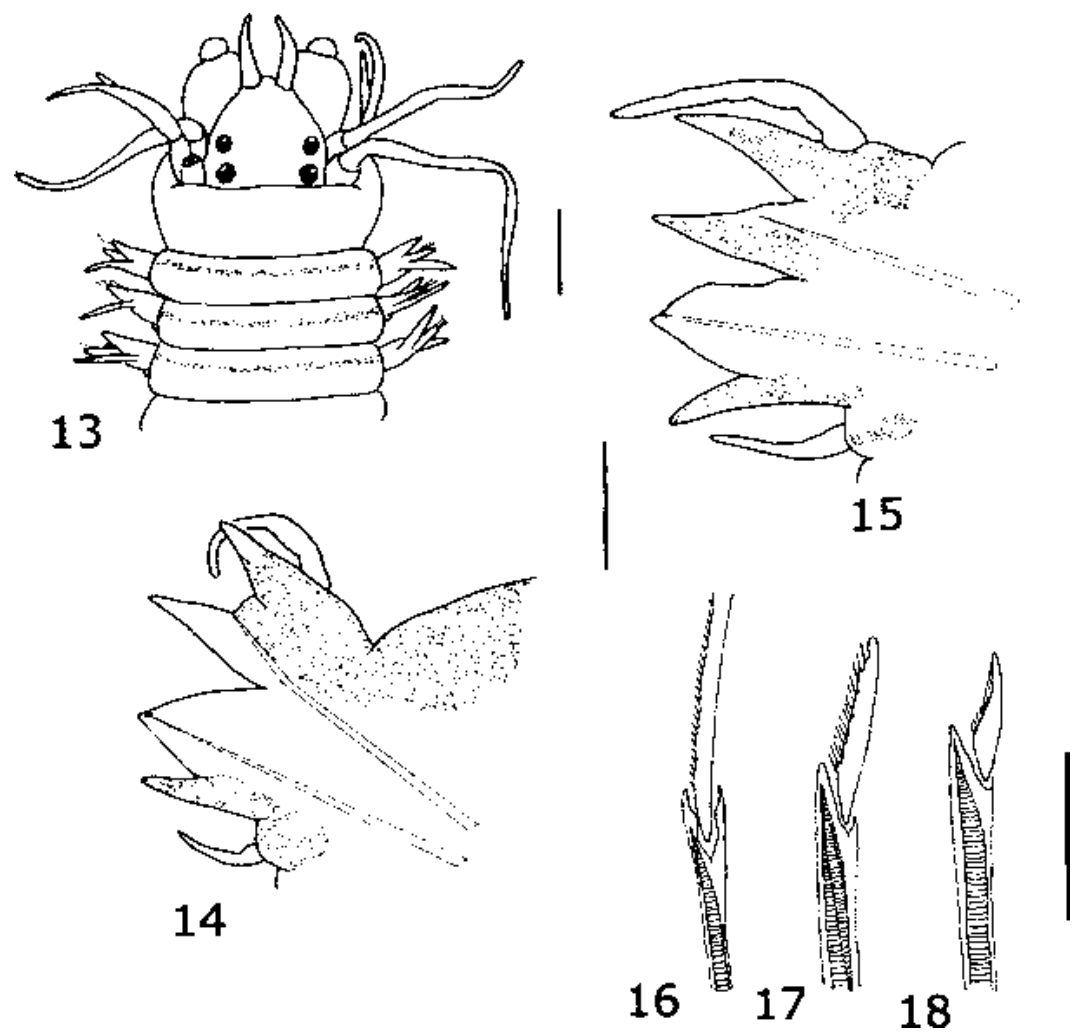

Figs. 13-18. Ceratonereis hircinicola (Eisig, 1870), Tambaú, Paraíba: 13, região anterior, vista dorsal; 14, parapódio 16, vista anterior; 15 , parapódio 57 , vista anterior; 16 , cerda espinígera homogonfa; 17,18, cerda heterogonfa falcígera. Escalas: $0,5 \mathrm{~mm}$, fig. $13 ; 0,1 \mathrm{~mm}$, figs. 14,$15 ; 0,05 \mathrm{~mm}$, figs. 16-18.

Habitat. Em recifes coralinos.

Distribuição. Oceano Índico, Madagáscar; Atlântico Oriental, Mar Mediterrâneo; Atlântico Ocidental, Brasil, Paraíba.

Material examinado. BRASIL, Paraíba: João Pessoa, Tambaú (MCEM 1127-1130, 7 exs.).

\section{Ceratonereis singularis Treadwell, 1929}

(Figs. 19-26)

Ceratonereis singularis Treadwell, 1929:1, figs. 1-8; Perkins, 1980:17, figs. 7-10.

Comentários. Ceratonereis singularis faz parte do grupo de espécies similares a $C$. mirabilis, caracterizado por cerdas falcígeras bidentadas, prostômio fendido e longos cirros dorsais (Perkins, 1980). Difere de C. excisa, descrita com base em exemplares da costa brasileira que também pertence a este grupo, por ter lígulas notopodiais posteriores. Apresenta o prostômio duas vezes mais largo do que longo e antenas foliáceas, largas e 


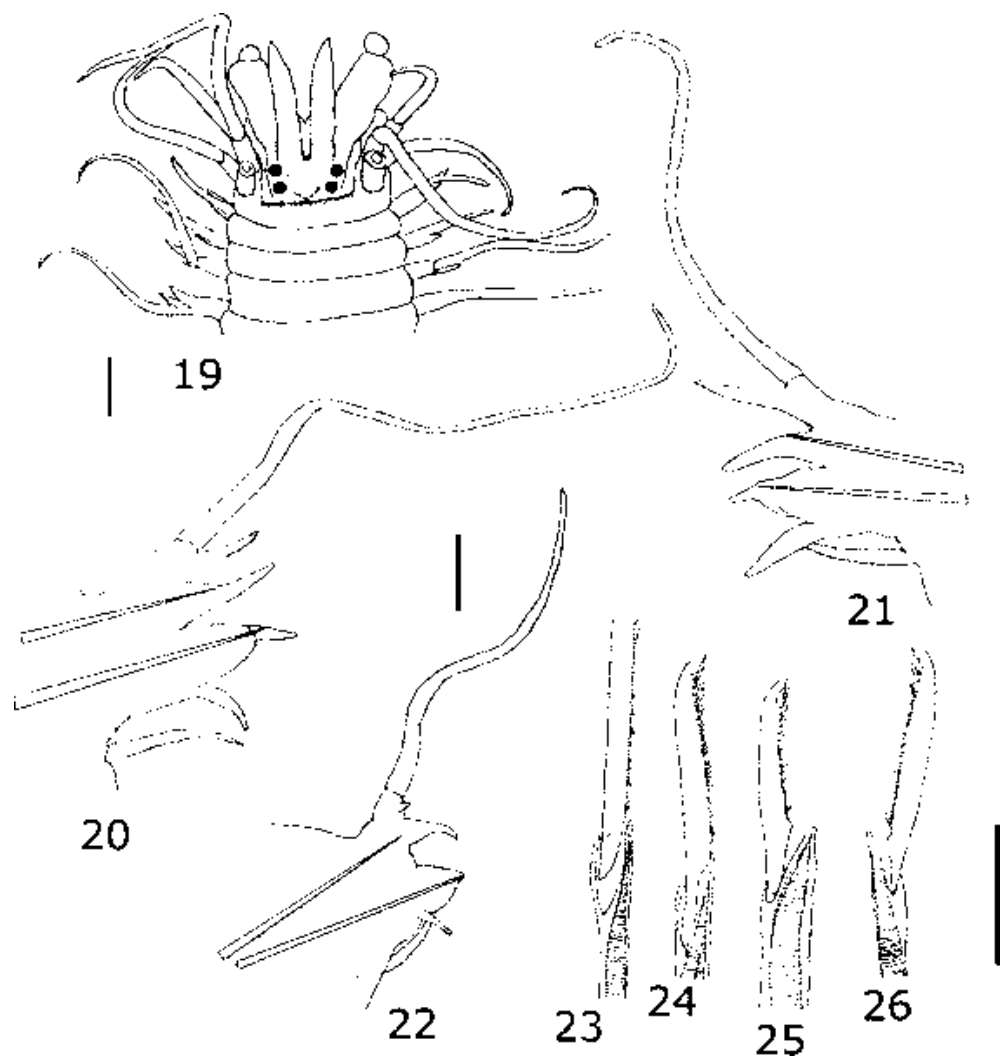

Figs 19-26. Ceratonereis singularis Treadwell, 1929, Maceió, Alagoas: 19, Região anterior, vista dorsal; 20, parapódio 5, vista anterior; 21, parapódio 18, vista posterior; 22, parapódio 59, vista anterior; 23 , cerda espinígera sesquigonfa; 24 , cerda falcígera sesquigonfa; 25,26 , cerda falcígera heterogonfa. Escalas: 0,5 mm, fig. 19, 23-26; 0,1 mm, figs. 20-22.

de comprimento equivalente à largura do prostômio, características marcantes que diferenciam C. singularis das demais espécies co-genéricas referidas para a costa brasileira.

PERKINs (1980) descreveu pequenas variações nos espécimes provenientes do Atlântico e àqueles do Pacífico. Os exemplares da costa nordeste assemelham-se aos descritos do Golfo do México, mas o prostômio apresenta-se um pouco mais largo que no material examinado por PERKINS (1980).

Habitat. Em entre marés e na plataforma continental, até $14 \mathrm{~m}$ de profundidade.

Distribuição. A espécie tem distribuição anfi-americana e os registros limitavam sua distribuição, no Atlântico Ocidental, até a Colômbia. Os registros aqui obtidos ampliam a distribuição da espécie cerca de $20^{\circ}$ para o sul. Atlântico Ocidental: Carolina do Norte, sudeste da Flórida, Golfo do México, Colômbia, região Nordeste do Brasil; Oceano Pacífico Oriental: México ao Panamá.

Material examinado. BRASIL, Maranhão: São Luís (MCEM 1152, 1 ex.); Paraíba: João Pessoa, Tambaú (MCEM 1139-1154, 18 exs.); Alagoas: Maceió (MCEM 1151, 2 exs.). 

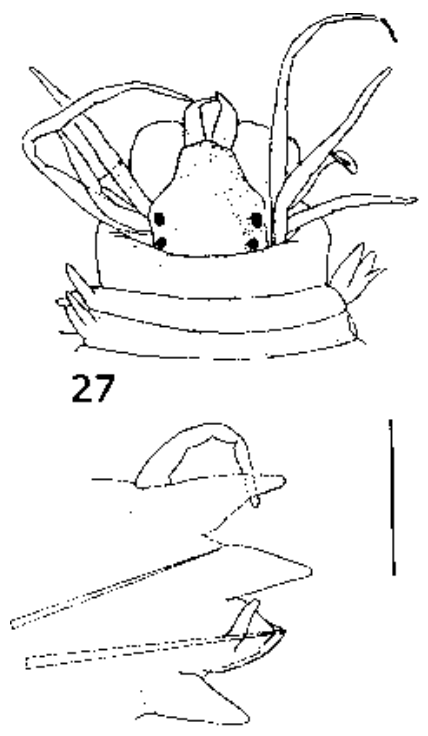

29
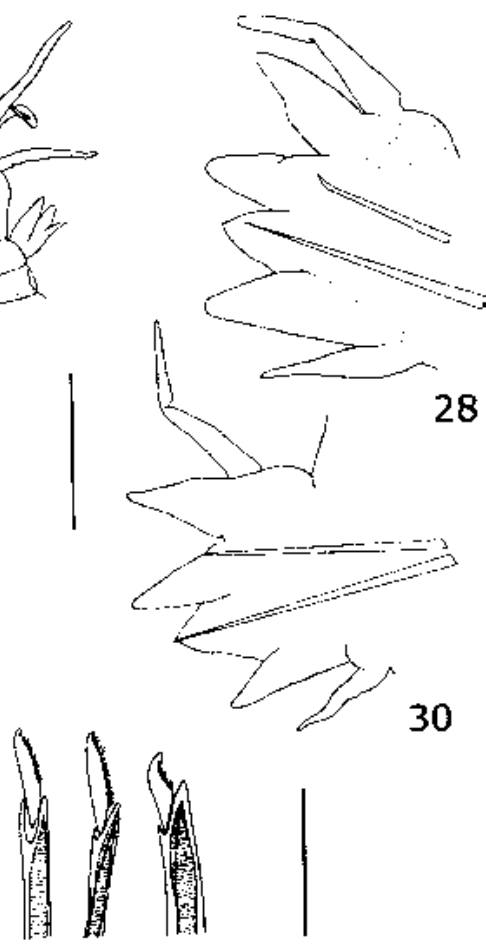

3132
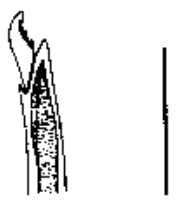

28

Figs. 27-33. Ceratonereis vittata Langerhans, 1884, Paraíba: 27, Região anterior, vista dorsal; 28, parapódio 6, vista posterior; 29, parapódio 20, vista posterior; 30, parapódio 35, vista posterior; 31 , cerda falcígera homogonfa; 32 , cerda falcígera heterogonfa; 33, cerda falcígera heterogonfa com tendão terminal. Escalas: 0,5 mm, figs. 27-30; 0,05 mm, figs. 31-33.

\section{Ceratonereis vitatta Langerhans, 1884}

(Figs. 27-33)

Ceratonereis vittata LANGerhans, 1884:254, lam. XV, fig. 12.

Comentários. O material é referido a $C$. vittata, embora neurocerdas espinígeras heterogonfas não tenham sido visualizadas, devido à ausência de paragnatas nas áreas II da probóscide. Nas demais espécies co-genéricas registradas na costa brasileira, com a região anterior do prostômio inteira e lígula notopodial, as paragnatas estão presentes nas áreas II.

Habitat. Na plataforma continental, a $26 \mathrm{~m}$ de profundidade, em substrato calcário.

Distribuição. Atlântico Oriental: Ilhas Açores, Madeira, Canárias, Camarões, Ilhas Príncipes, São Tomé, Ilhas de Rolas, Guiné Equatorial; Atlântico Ocidental: Paraíba e Ilhas Malvinas.

Material Examinado. BRASIL, Paraíba: Projeto Algas (Est. 53d, MCEM 1131, 1 ex.). 


\section{Nereis Linnaeus, 1758}

Chave para as espécies de Nereis da costa nordeste do Brasil.

1. Cerdas notopodiais falcígeras homogonfas sem dentes

Cerdas notopodiais falcígeras homogonfas com dentes pouco distintos ou conspicuamente bi, tri- ou tetradentadas 3

2. Área V sem paragnatas; áreas VII-VIII : 5 paragnatas N. riisei Grübe, 1857 Área V com uma paragnata cônica robusta; áreas VII-VIII: 2,3 fileiras irregulares de paragnatas, com fileira distal de paragnatas intercaladas N. oligohalina (Rioja, 1946)

3. Notocerdas homogonfas com dentes pouco distintos; lígulas notopodiais ausentes em parapódios posteriores N. serrata sp. nov.

Notocerdas homogonfas bi- ou tridentadas; lígulas notopodiais presentes, bem desenvolvidas ou vestigiais, em parapódios posteriores

4. Lígulas notopodiais ausentes ou vestigiais em parapódios posteriores. Notocerdas falcígeras homogonfas bidentadas N. falcaria (Willey, 1905)

Lígulas notopodiais nos parapódios posteriores. Notocerdas falcígeras homogonfas não denteadas N. pseudomoniliformis sp. nov.

\section{Nereis riisei Grübe, 1857}

(Figs. 34-40)

Nereis riisei GrüBe, 1857:162; Hartman, 1951:46; Nonato \& Luna, 1970:70, fig. 15; Liñero-Arana \& VASQuez, 1979:6, figs. 36a-g, lâm. 6,7; TAYLOR, 1984:31, figs. 31-35.

Nereis glandulata Hoagland, 1919:575, est. 30, figs. 1-6.

Comentários. Nereis riise i é facilmente identificada pela distribuição das paragnatas nas áreas VII-VIII. A espécie é bastante comum na costa brasileira e espécimes epítocos são facilmente coletados durante pesca noturna.

Variação. Alguns exemplares apresentam os palpos e o peristômio mais longos do que a maioria dos espécimes analisados. Nos mesmos exemplares, a borda anterior do prostômio se retrai em duas concavidades localizadas abaixo dos olhos. A pigmentação no prostômio na região dorso-lateral também variou, com exemplares dotados de pigmentação conspícua e outros completamente despigmentados; lígulas e lóbulos notoe neuropodiais apresentam-se mais ou menos pigmentados. É provável que algumas dessas variações devam-se ao processamento das amostras, como aplicação ou não de anestésicos e conservação.

Habitat. Em região entre marés de estuários, praias rochosas, com areia grossa, e na plataforma continental.

Distribuição. Atlântico Ocidental: águas temperadas e tropicais.

Material examinado. BRASIL, Maranhão: São Luís, Praia do Olho de Porco (MCEM 1082, 1089, 2 exs.); Piauí: Praia Luís Corrêa (MCEM 1055, 2 exs.); Praia do Coqueiro (MCEM 1088, 1 ex.); Ceará: Fortaleza (MCEM 1044, 1 ex.); Aracati (MCEM 1056, 1 ex.); Rio Grande do Norte: Natal (MCEM 1043, 4 exs.; MCEM 1083, 2 exs.); Búzios (MCEM 1045, 1 ex.); Tibaú do Sul (MCEM 1052, 1 ex.); Nísia Floresta (MCEM 1053, 1 ex.); Barra do Cunhaú (MCEM 1054, 1 ex.); Paraíba: Penha (MCEM 1049, 2 exs.); João Pessoa, Tambaú (MCEM 1060, 1062, 2 exs.); Cabo Branco (MCEM 1059, 1061, 1063, 3 exs.); Projeto Algas (MCEM 1064, 1085, 23 exs.); Pernambuco: Recife (MCEM 1046, 1 ex.); Praia dos Carneiros (MCEM 1058, 1 ex.); Bahia: Baía de Todos os 


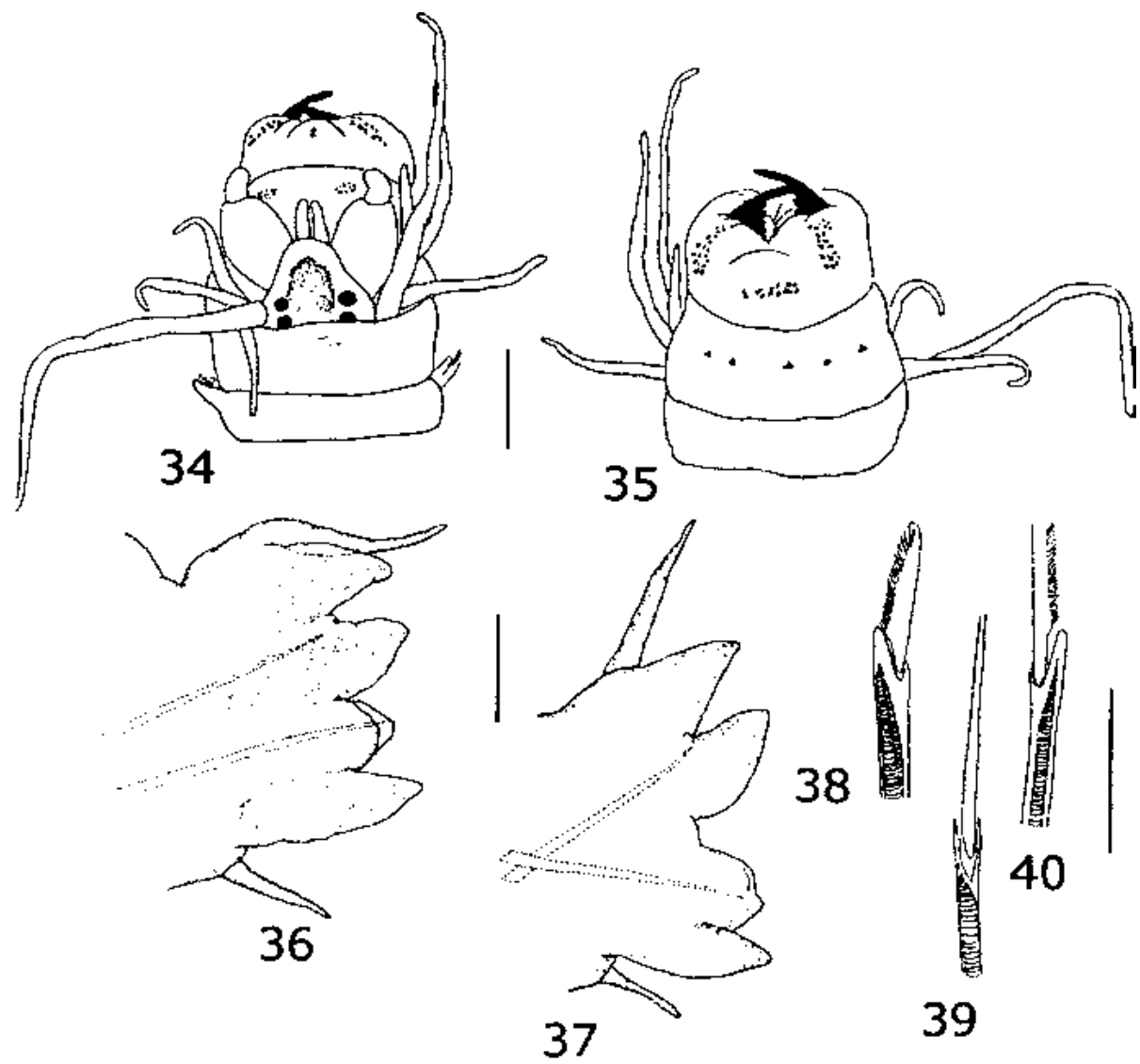

Figs. 34-40. Nereis riisei Grübe, 1857, Baía de Todos os Santos, Bahia: 34, região anterior com probóscide evertida, vista dorsal; 35, probóscide, vista ventral; 36, parapódio 29, vista posterior; 37, parapódio 80, vista posterior; 38 , cerda falcígera heterogonfa; 39 , cerda espinígera homogonfa; 40 , cerda espinígera heterogonfa. Escalas: $1,0 \mathrm{~mm}$, fig. $34 ; 0,1 \mathrm{~mm}$, figs. $35-37 ; 0,05 \mathrm{~mm}$, figs. 38-40.

Santos (MCEM 1037-1142, 8 exs.); Abrolhos (MCEM 1047, 1050, 2 exs.; MCEM 1079, 2 exs.); Ilhéus (MCEM 1051, 1 ex.); Cumuruxatiba (MCEM 1048, 3 exs.; MCEM 1078, 2 exs.; MCEM 1080, 1 ex.); Sta. Cruz de Cabrália (MCEM 1077, 1 ex.); Alagoas: Pontal do Peba (MCEM 1057, 2 exs.).

\section{Nereis falcaria (Willey, 1905)}

(Figs. 41-46)

Ceratonereis falcaria WilLEY, 1905:272, pl. 4, fig. 89.

Nereis kauderni Fauvel, 1921:9; figs. 1-7; Rullier \& Amoureux, 1979:167.

Nereis (Nereis) falcaria; DAY, 1967:309, figs. 14.5 h-m.

Comentários. As características diagnósticas da espécie, como as robustas notocerdas falcígeras homogonfas e as paragnatas pálidas de aspecto delicado e de difícil visualização, são perfeitamente verificáveis nos espécimes analisados. Difere de 

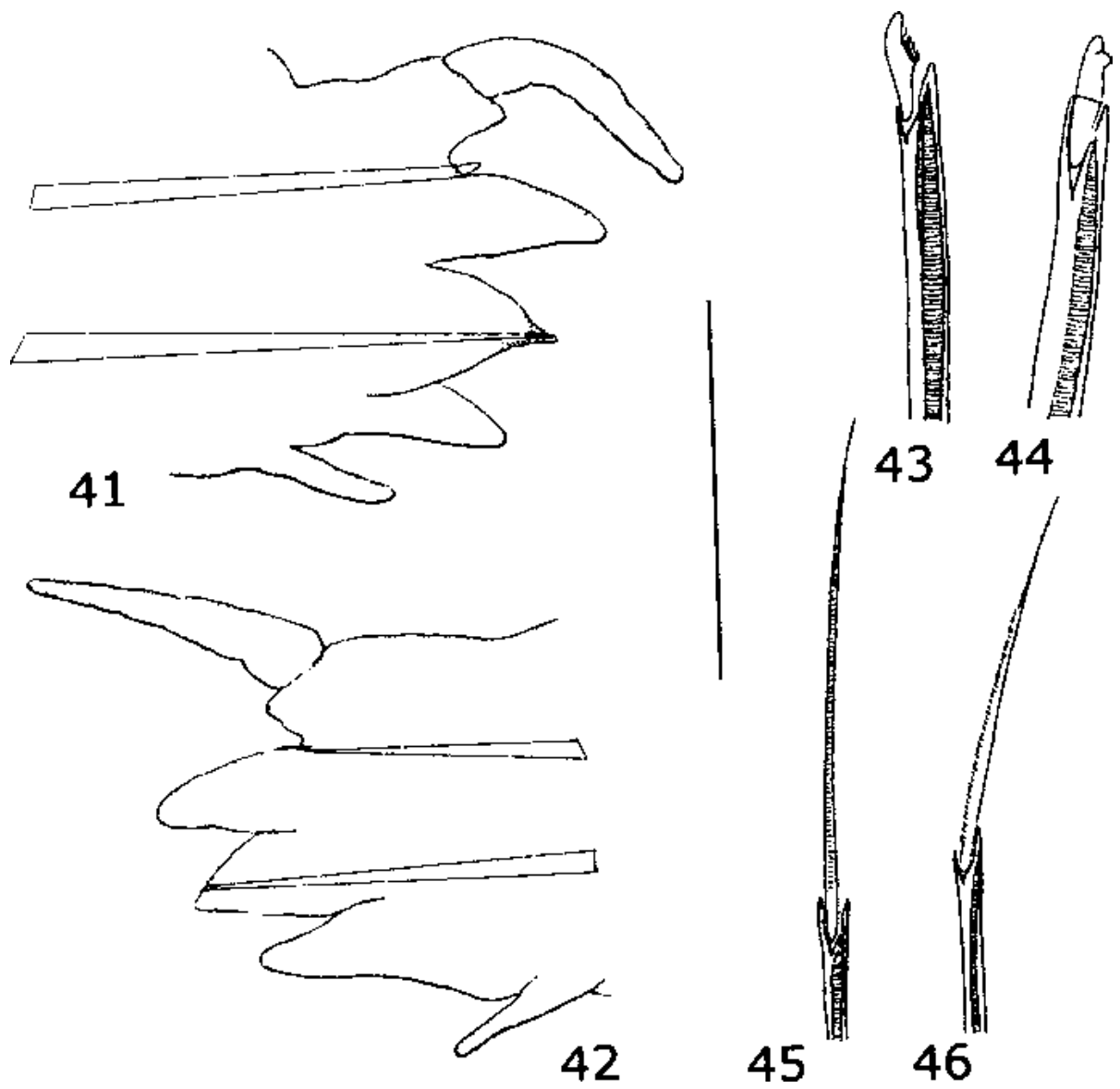

Figs. 41-46. Nereis falcaria (Willey, 1905), Atol das Rocas, Rio Grande do Norte: 41, parapódio anterior, vista posterior; 42, parapódio mediano, vista anterior; 43, cerda falcígera heterogonfa; 44, cerda falcígera homogonfa bidentada; 45 , cerda espinígera homogonfa; 46 , cerda espinígera heterogonfa. Escalas: 0,1 mm, figs. 41-46.

Nereis jacksoni Kinberg, 1866, espécie similar, por apresentar prostômio fendido anteriormente, pigmentação e paragnatas com arranjo distinto.

Segundo DAY (1967), os cirros notopodiais anteriores são inseridos na base de uma lígula notopodial desenvolvida. No material do nordeste, os parapódios anteriores apresentam cirros inseridos subterminalmente na lígula notopodial desenvolvida, tal como descritos por FaUvel (1921) sob o nome Nereis kauderni, que DaY (1967) considerou idêntica a Nereis falcaria.

Habitat. Poças de maré com areia fina.

Distribuição. Circumtropical .

Material examinado. BRASIL, Rio Grande do Norte: Atol das Rocas (MCEM 1123, 1124, 2 exs.). 


\section{Nereis serrata sp. nov.}

(Figs. 47-56)

Etimologia. $\mathrm{O}$ adjetivo específico refere-se à borda da lâmina nas cerdas homogonfas falcígeras, que apresentam dentes pouco distintos, formando uma serrilha delicada (serrata $=$ parecido com uma serrilha).

Diagnose. Nereis serrata caracteriza-se pela margem anterior dos 12-13 primeiros setígeros tipicamente dobrada sobre o setígero anterior (fig. 47); ausência de lígulas notopodiais posteriores (fig. 55) e notocerdas falcígeras homogonfas com denteação pouco distinta (figs. 54-56).

Descrição. Holótipo: espécime completo com 56 setígeros e $15 \mathrm{~mm}$ de comprimento total, $750 \mu \mathrm{m}$ de largura sem os parapódios e $1125 \mu \mathrm{m}$ com os parapódios. Corpo cilíndrico; coloração amarelada; 12-13 primeiros setígeros com a borda anterior formando uma pequena dobra sobre o setígero imediatamente anterior. Prostômio quadrangular; um par de antenas frontais, cirriformes, mais longas que os palpos; dois pares de olhos negros, dispostos em retângulo na metade posterior do prostômio, com o par posterior recoberto por dobra do peristômio; um par de palpos, com palpóforos cilíndricos e palpóstilos esféricos, pequenos; peristômio uma vez e meio mais longo que o setígero 1; quatro pares de cirros anteriores, o mais longo atinge a margem anterior do setígero 1 (fig. 47). Delimitação entre os palpos e prostômio tênue e difícil de ser visualizada, dando a impressão de uma estrutura única. Probóscide com paragnatas de difícil visualização nos dois anéis: área I: 0; áreas II: 0-2 paragnatas; área III: 8 paragnatas em elipse; áreas IV: 5 paragnatas em 1-2 fileiras em arco; área V: 0; áreas VI: 1-2; áreas VII-VIII: 10 paragnatas pequenas em fileira única. Cirro dorsal cônico, mais longo que a lígula notopodial ao longo do corpo. Lígula notopodial cônica presente a partir do setígero 4, diminuindo e tornando-se vestigial nos setígeros 22-23, onde desaparece (figs. 48, 49). Lóbulo neuropodial acicular triangular, mais curto que o notopodial; lígula neuropodial cônica mais longa que o lóbulo na maior parte dos parapódios, diminuindo apenas nos parapódios próximos ao pigídeo (figs. 4851). Notocerdas espinígeras homogonfas até o setígero 17, a partir daí são substituídas por homogonfas falcígeras com dentes pouco distintos (figs. 52-54); neurocerdas supraaciculares espinígeras homogonfas e falcígeras heterogonfas; neurocerdas subaciculares espinígeras e falcígeras heterogonfas (figs. 55, 56). Acículas âmbar. Pigídio com dois uritos curtos, atingindo o comprimento de 3 setígeros.

Habitat. Em substrato calcário de plataforma, entre 12 e $21 \mathrm{~m}$.

Distribuição. Plataforma continental brasileira, Ceará.

Comentários. Hutchings \& TURVEY (1982) revisaram os nereidídeos da Austrália e agruparam diversas espécies do gênero Nereis, muito similares, com base na denteação das cerdas falcígeras homogonfas, na distribuição das paragnatas nas áreas VII-VIII e na presença ou ausência de lígulas notopodiais posteriores. Nereis serrata é tentativamente referida ao grupo descrito por HUTCHINGS \& TURVEY (1982). Entre as espécies deste grupo, N. falcaria, N. jacksoni Kinberg, 1866 e N. panamensis Fauchald, 1977 são as mais similares a Nereis serrata.

Nereis falcaria difere de $N$. serrata pelo prostômio fendido e cerdas falcígeras homogonfas conspicuamente bidentadas. Nereis jacksoni apresenta lígulas notopodiais posteriores e numerosas paragnatas nas áreas VII-VIII distribuídas em duas fileiras 


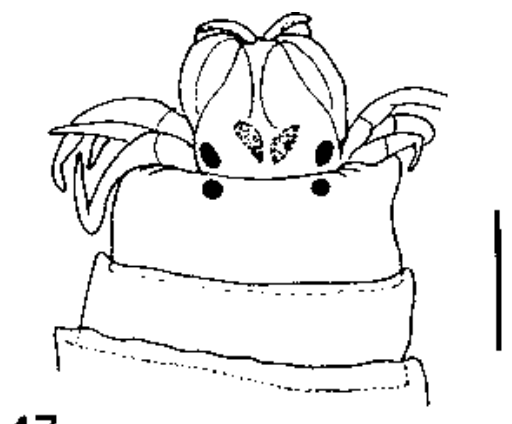

47

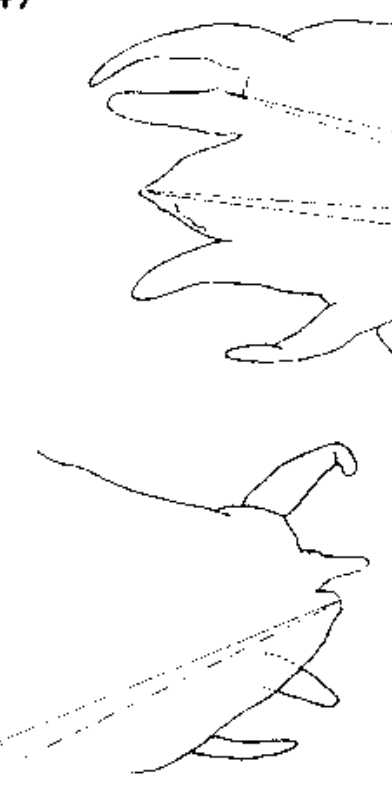

51
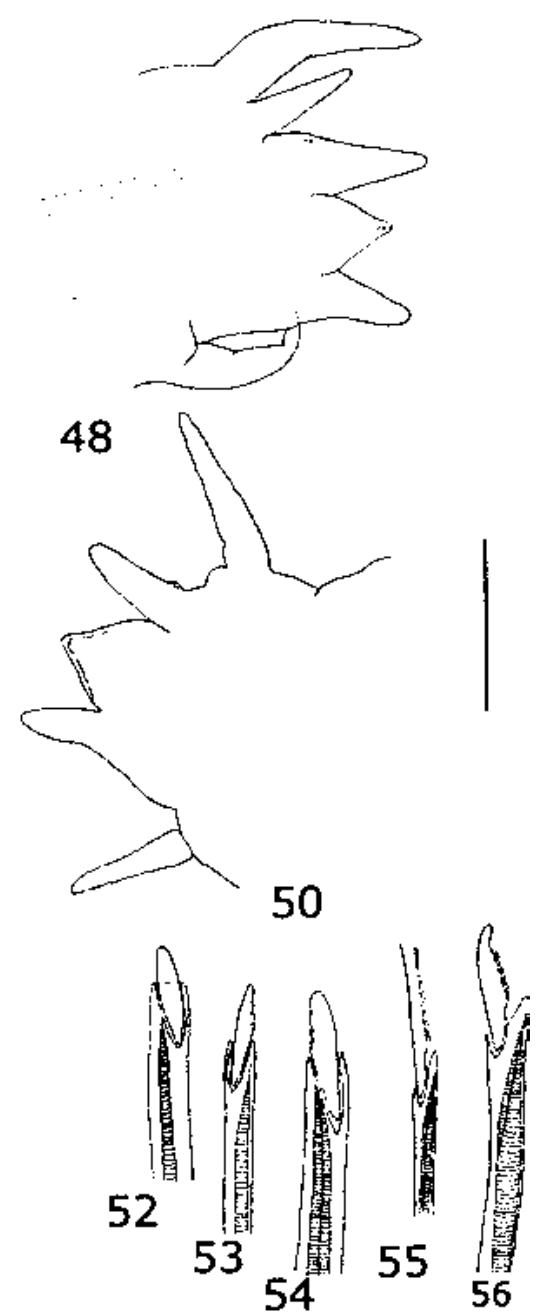

Figs. 47-56. Nereis serrata sp. nov., holótipo, Plataforma Continental, Ceará: 47, região anterior, vista dorsal; 48, parapódio 6, vista posterior; 49, parapódio 20, vista posterior; 50, parapódio 33, vista posterior; 51 , parapódio 57 , vista anterior; $52-54$, cerdas falcígeras homogonfas; 55 , cerda espínigera heterogonfa; 56 cerda falcígera heterogonfa. Escalas: $0,2 \mathrm{~mm}$, fig. $47 ; 0,1 \mathrm{~mm}$, figs. $48-50 ; 0,05 \mathrm{~mm}$, figs. 52-56.

irregulares. Nereis panamensis assemelha-se a $N$. serrata pela distribuição das paragnatas e morfologia parapodial; no entanto, tem a margem anterior do prostômio fendida e cerdas falcígeras homogonfas distintamente denteadas.

Material-tipo. BRASIL, Ceará: Projeto GEOCOSTA I, holótipo (MCEM 1117); parátipos (MCEM 1116, 4 exs.; MCEM 1118-1121, 10 exs.). 


\section{Nereis pseudomoniliformis sp. nov.}

(Figs. 57-63)

Etimologia. O nome específico refere-se à aparência moniliforme dos cirros anteriores (moniliformis do latim, que apresenta estrangulamentos e dilatações, com forma de colar).

Diagnose. Caracteriza-se pela aparência moniliforme dos cirros anteriores (fig. 57); paragnatas cônicas em ambos os anéis; área I:0-2 paragnatas pequenas; áreas II:11 pequenas em fileira oblíqua; área III:1; áreas IV:9-11 paragnatas, algumas em círculo e um número menor em fileira; área V:0; áreas VI:2-3 paragnatas em fileira horizontal sobre lobos em forma de almofada; áreas VII-VIII:2-3 fileiras irregulares com paragnatas pequenas; lígula notopodial em parapódios posteriores (fig. 60); notocerdas homogonfas bi- e tridentadas (figs. 61, 62).

Descrição. Holótipo: espécime completo com 47 setígeros e $7 \mathrm{~mm}$ de comprimento total, e $500 \mu \mathrm{m}$ de largura sem os parapódios e $750 \mu \mathrm{m}$ com os parapódios. Espécimes delicados, amarelo-claros. Prostômio mais longo que largo; um par de antenas frontais, cirriformes, ligeiramente mais longas que os palpos; dois pares de olhos, negros, circulares, dispostos em retângulo na metade posterior do prostômio, o par anterior é maior que o posterior; peristômio uma vez e meio mais longo que o setígero 1; quatro pares de cirros anteriores, o mais longo atingindo o setígero 4 . Antenas e cirros anteriores com constrições alternadas e incompletas, o que lhes dá uma aparência anelada (fig. 57). Paragnatas cônicas em ambos os anéis: área I: 0-2 paragnatas pequenas; áreas II:11 paragnatas pequenas em fileira oblíqua; área III:1?; áreas IV:9-11 paragnatas, algumas em círculo e um número menor em fileira; área V:0; áreas VI:2-3 paragnatas em fileira horizontal sobre lobos em forma de almofada; áreas VII-VIII: 2-3 fileiras irregulares com paragnatas pequenas. Em alguns espécimes as paragnatas são pouco visíveis. Mandíbulas transparentes, com aspecto delicado. Cirro dorsal cônico com inserção basal e mais longo que a lígula notopodial em todos os parapódios. Lígulas e lóbulos notopodiais cônicos, divergentes, aproximadamente do mesmo tamanho na região anterior do corpo com a lígula notopodial, mais curta que o lóbulo em parapódios medianos e posteriores (figs. 58, 59). Lóbulo neuropodial pré-cerdal triangular do mesmo comprimento que o lóbulo pós-cerdal. Lígula neuropodial cônica, tão longa quanto o lóbulo neuropodial ao longo de todo o corpo. Cirro ventral cônico, mais curto que a lígula neuropodial ao longo de todo o corpo (figs. 58-60). Notocerdas espinígeras homogonfas até o setígero 17, onde são substituídas por 1-3 cerdas falcígeras homogonfas com três, quatro e até cinco dentes (figs. 61, 62). Neurocerdas supra-aciculares espinígeras homogonfas e falcígeras heterogonfas; neurocerdas subaciculares falcígeras heterogonfas (fig. 63). Pigídio com uritos filiformes que atingem o comprimento de 4-7 setígeros.

Habitat. Região sublitoral estuarina, em substrato arenoso.

Distribuição. Brasil, Sergipe, Estuário do rio Piauí, sul do Estado.

Comentários. Nereis pseudomoniliformis assemelha-se a N. zonata Malmgren, 1867 na distribuição das paragnatas e pela morfologia parapodial, difere desta pelas cerdas notopodiais falcígeras homogonfas distintamente denteadas. Nereis zonata é amplamente referida para águas temperadas e frias do hemisfério norte. Nereis pseudomoniliformis assemelha-se a $N$. triangularis Hutchings \& Turvey, 1982, ocorrente na Austrália, pelas antenas e cirros anteriores com aparência anelada, lígulas notopodiais posteriores e 

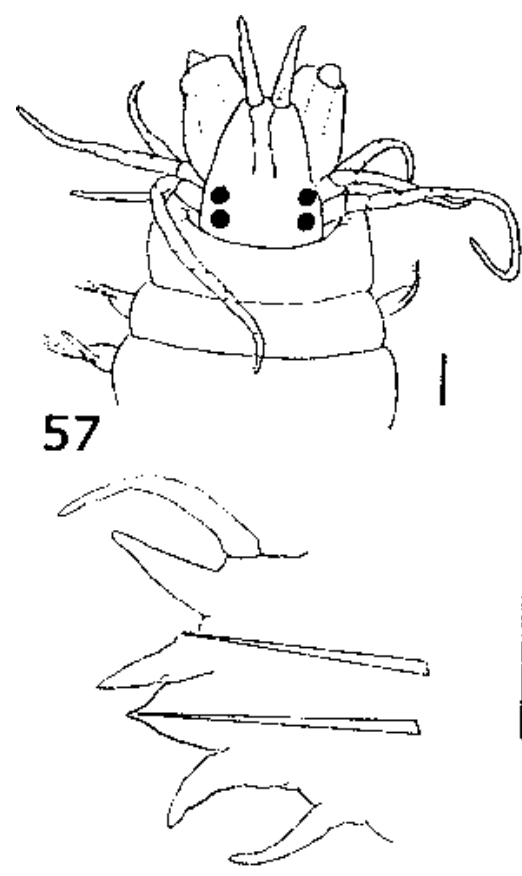

59

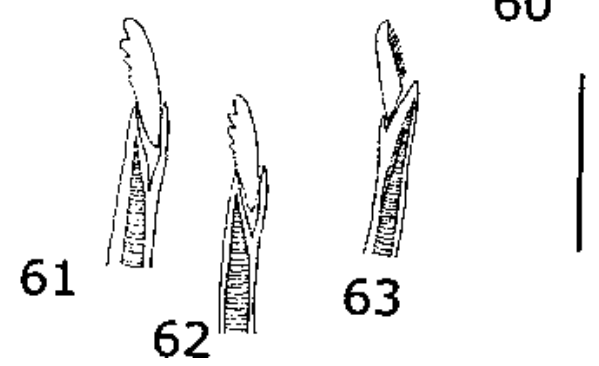

Figs. 57-63. Nereis pseudomoniliformis sp. nov., Rio Piauí, Sergipe, holótipo: 57, região anterior, vista dorsal; 58, parapódio 5, vista posterior; 59, parapódio 26, vista anterior; 60, parapódio 40, vista posterior, 61-62, cerdas falcígeras homogonfas; 63, cerda falcígera heterogonfa. Escalas: 0,1 mm, fig. 57 ; $0,2 \mathrm{~mm}$, figs. $58-60 ; 0,05 \mathrm{~mm}$, figs. 61-63.

paragnatas nas áreas VII-VIII dispostas em fileira única; difere desta pelas lígulas notopodiais posteriores conspicuamente menores que os lóbulos notopodiais, lígulas neuropodiais posteriores menores que os lóbulos e pelo menor número de paragnatas em todas as áreas da probóscide. Separa-se de Nereis trifasciata Grübe, 1878 pelas cerdas notopodiais falcígeras lisas e pela distribuição das paragnatas nas áreas VII-VIII.

Material-tipo. BRASIL, Sergipe: Estuário do Rio Piauí, holótipo (MCEM 1104); parátipos (MCEM 1105-1115, 56 exs.). 


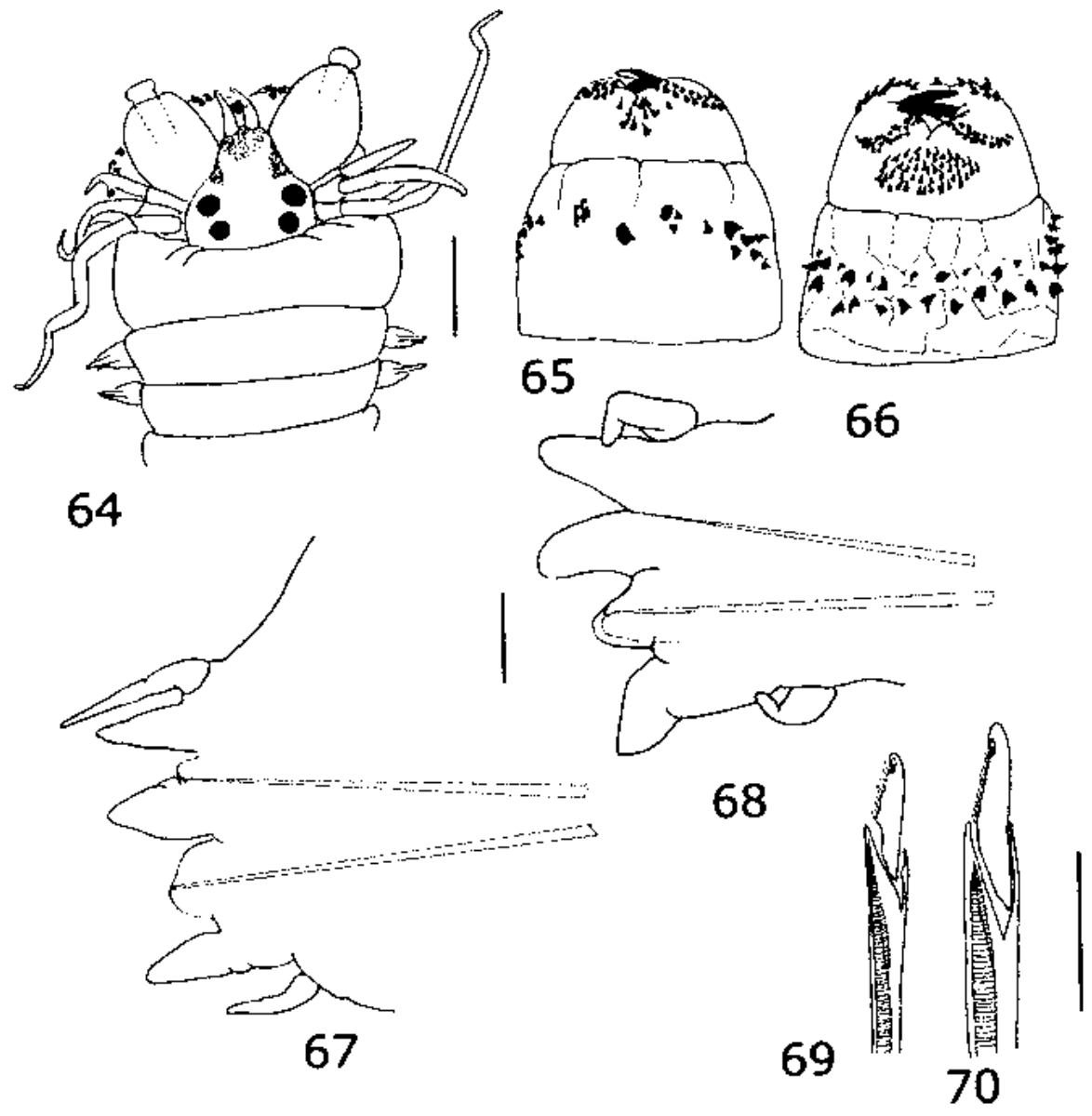

Figs. 64-70. Nereis oligohalina (Rioja, 1946), Baía de Todos os Santos, Bahia: 64, região anterior, vista dorsal; 65, probóscide, vista dorsal; 66, probóscide, vista ventral; 67, parapódio 3, vista anterior; 68, parapódio 27, vista anterior; 69, cerda falcígera heterogonfa; 70, cerda falcígera homogonfa;. Escalas: $1,0 \mathrm{~mm}$, fig. $64 ; 0,1 \mathrm{~mm}$, figs. $65-68 ; 0,05 \mathrm{~mm}$, figs. 69,70 .

\section{Nereis oligohalina (Rioja, 1946)}

(Figs. 64-70)

Neanthes oligohalina RIOJA, 1946:207; figs. 3-6.

Nereis oligohalina; RioJA, 1947:529; HARTMAN, 1951:46.

Comentários. O material proveniente da Bahia corresponde à descrição de RıOJA $(1946,1947)$ na forma da lígula neuropodial dos parapódios posteriores. Espécimes procedentes do Maranhão não apresentam lígulas neuropodiais tão desenvolvidas, assim 
como os exemplares identificados no Paraná (obs. pess.). Devido à constância da pigmentação prostomial e a distribuição das paragnatas, a variação neuropodial é considerada como uma diferenciação populacional. Além da forma diferente da lígula, os exemplares do Maranhão são menores que os da Bahia; contudo o número e distribuição das paragnatas, cerdas e pigmentação prostomial são constantes e idênticos ao referido para a espécie.

Nereis oligohalina foi considerada idêntica a N. occidentalis Hartman, 1945 por PetTiBone (1956), para quem a diferença no número de paragnatas seria uma simples variação populacional. Os exemplares descritos por PetTiBOne (1956), além da diferença no número de paragnatas em outras áreas, não apresentam as 7 pequenas paragnatas intercaladas na fileira distal de paragnatas mais robustas nas áreas VII-VIII, têm a lígula neuropodial de parapódios posteriores aproximadamente do mesmo comprimento do lóbulo neuropodial e cirros dorsais duas vezes maiores que a lígula notopodial; em N. oligohalina, os cirros são ligeiramente maiores que a lígula notopodial. Com base no número de paragnatas e tamanho dos cirros e lígulas, DAY (1973) considerou $N$. occidentalis idêntica a $N$. falsa Quatrefages, 1865. Os exemplares do nordeste do Brasil são aqui referidos a $N$. oligohalina, considerada espécie válida até que um estudo de exemplares provenientes de todo o Atlântico Ocidental seja realizado.

Habitat. Em região entre marés de estuários e em praia areno-lodosa.

Distribuição. Atlântico Ocidental: em áreas estuarinas do Golfo do México; norte e nordeste do Brasil (Maranhão, Rio Grande do Norte, Alagoas e Bahia); sul do Brasil (Paraná).

Material examinado. BRASIL, Maranhão: São Luís (MCEM 1092-1100, 22 exs.); Rio Grande do Norte: Praia da Pipa (MCEM 1091, 1 ex.); Alagoas: Paripueira (MCEM 1090, 1 ex.); Bahia: Baía de Todos os Santos (MCEM 1102, 1103, 2 exs.).

Agradecimentos. À CAPES, pelo apoio financeiro ao projeto. A todos que cederam nereidídeos para este estudo: Verônica Oliveira (Universidade Federal do Maranhão), Wilson Franklin (Universidade Federal do Ceará), Rosângela Gondim (Universidade Federal do Rio Grande do Norte), Martin Christoffersen e Elineí Araújo (Universidade Federal da Paraíba), Bety Rose (Universidade Federal Rural de Pernambuco), Tereza Callado (Universidade Federal de Alagoas), Maria Auxiliadora Santos, Erminda Couto, Wellington Sousa e Denise Santos (Universidade Federal de Sergipe), Lêda Santa-Isabel (Universidade Federal da Bahia), Paulo César de Paiva (Universidade Federal Rural do Rio de Janeiro) e Sérgio Netto (UNISUL, Santa Catarina).

\section{REFERÊNCIAS BIBLIOGRÁFICAS}

Augener, H. 1913. Polychaeta I, Errantia. In: Michaelson, W. \& Hartmeyer, R. eds. Die fauna Australiens. Jena. v. 4, p. 65-304.

DAY, J. H. 1967. A monograph on the Polychaeta of southern Africa: Part I Errantia. London, British Museum. 374p.

1973. New polychaeta from Beaufort, with a key to all species recorded from North Carolina. NOAA Tech. Rep., Washington, 375:1-140.

EIsIG, H. 1870. Nereis hircinicola (nova species). Z. wiss. Zool, Leipzig, 20:103-105.

Fauvel, P. 1921. Annélides polychètes de Madagascar, du museum R. d' Histoire naturelle recueillieis par M. le Dr. W. Kaudern en 1912. Ark. Zool., Stockholm, 13(24):1-32.

1923. Polychétes errantes. Faune Fr., Paris, 5:1-488.

GrüBE, A. E. 1857. Annulata Orstediana. Enumeratio Annulatorum, quac in itinere per Indiam occidentalem et Americam centralem annis 1845-1848 suscepto legit cl. A. S. Orsted, adjectis speciebus nonnullis a cl. H. Kroyero in itinere ad Americam meridionalem collectis. Vidensk. Meddr dansk naturh. Foren., Kopenhagen, 2:158-166. 
1874. Die familie Lycoriden und die aufstellung von gruppen in der Gatung Nereis. Ges. Vaterl. Kult., Breslau, 51:56-73.

Hansen, A. 1882. Recherches sur les Annélides recueillies par M. le Professeur Édouard Van Beneden pendant son voyage au Brésil et à la Plata. Mém. Acad. R. Sc. Belg., Bruxelles, 44:1-29.

HaRTMAn, O. 1948. The marine annelids erected by Kinberg, with some notes on some other types in the Swedish State Museum. Ark. Zool., Stockholm, 42A(1):1-137.

_. 1951. The littoral marine annelids of the Gulf of Mexico. Publ. Inst. Mar. Sci. Univ Tex., Port Aransas, 2:7-124.

Hartmann-SchröDer, G. 1985. Revision der Gattung Ceratonereis Kinberg (Nereididae, Polychaeta) (mit besonderer berucksichtigung der auten mit eingeschnittenem prostomium). Mitt. hamb. zool. Mus. Inst., Hamburg, 82:37-59.

Hoagland, R. A. 1919. Article XVI. Polychaetous annelids from Porto Rico, the Florida keys, and Bermuda. Bull. Am. Mus. nat. Hist., New York, 41:571-590.

Holme, N. A. \& McIntyre, A. D. 1971. Methods for the study of marine benthos. Oxford, Blackwell Scientific. 334p.

Hutchings, P. \& Turvey, S. P. 1982. The Nereididae of south Australia. Trans. R. Soc. S. Aust., Adelaide, 106:93-144.

IмалтмA, M. 1972. Review of the annelid worms of the family Nereidae of Japan, with descriptions of five new species or subspecies. Bull. Natl. Sci. Mus., Tokyo, 15(1):37-153.

Kingerg, J. 1866. Annulata nova. Öfvers. K. VetenskAkad. Förh., Stockholm, 22:167-179.

Lana, P. C. \& Sovierzoski, H. H. 1987. Neanthes bruaca sp. n. e Nereis broa sp. n. (Nereididae: Polychaeta) da costa sudeste do Brasil. Arq. Biol. Technol., Curitiba, 30(4):677-688.

Langerhans, P. 1884. Die Wurmfauna von Madeira. IV. Z. wiss. Zool., Leipzig, 40:247-285.

Liñero-Arana, I. \& Vasquez, G. R. 1979. Nereidae (Polychaeta, Errantia) del Golfo de Cariaco, Venezuela. Boln Inst. Oceanogr. Univ. Oriente, Cumana, 18(1/2):3-12.

Morgado, E. H.; Amaral, A. C. Z. et al. 1994. Intertidal sandy beaches polychaetes of São Sebastião Island, southern Brazil. Mém. Mus. natn. Hist. nat., Paris, 162:485-492.

Nonato, E. F. \& LunA, J. A. C. 1970. Anelídeos poliquetas do nordeste do Brasil : I. - poliquetas bentônicos da costa de Alagoas e Sergipe. Bolm Inst. Oceanogr. S. Paulo, São Paulo, 19(1):57-130.

Pagliosa, P. R. \& Lana, P. C. 2000. Population dynamics and secondary production of Nereis oligohalina (Nereididae, Polychaeta) from a subtropical marsh in southeast Brazil. Bull. Mar. Sci., Miami, 67(1):259-268.

Paiva, P. C. 1993. Anelídeos poliquetas da plataforma continental norte do Estado de São Paulo: I Padrões de densidade e diversidade específica. Bolm Inst. Oceanogr. S. Paulo, São Paulo, 41(1/ 2):69-80.

Perkins, T. H. 1980. Review of species referred to Ceratonereis mirabilis, and descriptions of new species of Ceratonereis, Nepthys, and Goniada (Polychaeta). Proc. Biol. Soc. Wash., Washington, 93(1):1-49.

Pettibone, M. H. 1956. Some polychaete worms of the families Hesionidae, Syllidae, and Nereidae from the east coast of North America, West Indies, and Gulf of Mexico. J. Wash. Acad. Sci., Washington, 46(9):281-294.

RioJA, E. 1946. Estudios anelidologicos XV. Nereidos de agua salobre de los esteros litoral del Golfo de Mexico. An. Inst. Biol. Univ. Méx., México, 17:205-214.

1947. Estudios anelidologicos XIX: observaciones sobre algunos nereidos de las costas de Mexico. An. Inst. Biol. Univ. Mexico, México, 18:527-535.

Rullier, F. \& Amoureux, L. 1979. Annélides polychètes. Résultats scientifiques des campagnes de la Calypso. Ann. Inst. Océanogr., Monaco, 55 (Suppl.):10-206.

Santa-Isabel, L. M.; Leão, Z. M. A. \& Peso-Aguiar, M. C. 2000. Polychaetes from the Guarajuba coral reefs, Bahia, Brazil. Bull. Mar. Sci., Miami, 67(1):645-654.

Taylor, J. L. 1984. Family Nereidae Johnston, 1845. In: Uebelacker, J. M. \& Johnson, P. G. Taxonomic guide to the polychaetes of the northern Gulf of Mexico. Mobile, Barry A. Vittor \& Associates. v. 5, p. 1- 42 .

Treadwell, A. L. 1929. New species of polychaetous annelids in the collections of the American Museum of Natural History from Porto Rico, Florida, Lower California, and British Somaliland. Am. Mus. Novit., New York, 392:1-13.

1932. New species of polychaetous annelids from Ilha de São Sebastião, together with a list of 
other annelids determined from Brasil. Revta Mus. paul., São Paulo, 17(2):13-22.

Willey, A. 1905. Report on the Polychaeta collected by Professor Herdman, at Ceylon, in 1902.

Suppl. Rep. Ceylon Pearl Oyster fish, Ceylon, 4:243-324.

Recebido em 22.10.2000; aceito em 08.11.2002

Iheringia, Sér. Zool., Porto Alegre, 93(1):5-22, 30 de março de 2003 